\title{
Hypoglossal Nerve Paresis Caused by Spontaneous Dissection of Kinked Internal Carotid Artery
}

\author{
-Case Report-
}

\author{
Yasushi MIZUMAKI, Shunro ENDO, Kazumasa YAMATANI, \\ Akira TAKAKU, and Eiji TSUKAMOTO* \\ Department of Neurosurgery, Toyama Medical and Pharmaceutical University, Toyama; \\ *Department of Neurosurgery, Tsukamoto Hospital, Toyama
}

\begin{abstract}
A 54-year-old male presented with pure left hypoglossal nerve paresis. Angiography and magnetic resonance (MR) imaging showed the characteristic findings of left internal carotid artery (ICA) dissection. He received aspirin and his symptoms gradually disappeared. Repeat angiography and MR imaging showed that the lesion had completely disappeared. This case supports the hypothesis that hypoglossal nerve paresis is due to nerve stretching and compression by intramural hematoma of the dissected ipsilateral ICA, and severe tortuosity of the ICA may be a related phenomenon.
\end{abstract}

Key words: hypoglossal nerve paresis, internal carotid artery, dissection, diagnosis

\section{Introduction}

Clinical symptoms of internal carotid artery (ICA) dissection include headache, focal cerebral ischemic symptoms, oculosympathetic nerve paresis, neck pain, syncope, and amaurosis fugax. ${ }^{5)}$ Hypoglossal nerve paresis is rare, with only a few cases reported. ${ }^{2,3,5,7,81}$ The mechanism of the paresis may be mechanical compression and/or stretching by enlargement of the ipsilateral ICA. ${ }^{3,4)}$ We present a case of ICA dissection with hypoglossal nerve paresis without other neurological symptoms and discuss the pathological mechanism.

\section{Case Report}

A 54-year-old male experienced sudden onset of headache on June 27,1995 , which eased but did not resolve. Ten days after onset, his tongue became uncoordinated and his speech was slurred. He had previously been well, with no history of head or neck trauma, hypertension, or heart disease, and no inflammatory signs.

Neurological examination on admission found the major abnormality was asymmetrical tongue position at rest and left hypoglossal nerve paresis (Fig. 1 upper). Gag reflex was normal and swallowing was intact. Palatal, lingual, buccal, and tongue sensation were normal. There was no evidence of oculosympathetic nerve paresis, long tract sign, or cerebellar deficit. Angiography showed tortuosity at the origin of the ICA and diffuse irregular narrowing of the external portion (Fig. 2 upper row). Magnetic resonance (MR) imaging showed a high intensity signal on $\mathrm{T}_{1^{-}}$and $\mathrm{T}_{2}$-weighted images suggesting intramural hematoma due to dissection inducing severe stenosis, corresponding to the angiographic findings (Fig. 3).

He was treated with aspirin, and his symptoms gradually improved and completely disappeared 6 weeks after onset (Fig. 1 lower). Repeat angiography showed that the diffuse narrowing previously observed had completely disappeared. However, the tortuosity at the origin of the ICA was observed more clearly and a change in the course of the ICA was also indicated (Fig. 2 lower row). Repeat MR imaging did not show any high intensity lesion in the arterial wall (Fig. 4). No abnormal intracranial lesions were identified. He was discharged without neurological deficit.

\section{Discussion}

In some reported cases hypoglossal nerve paresis

Received April 1, 1996; Accepted January 6, 1998 


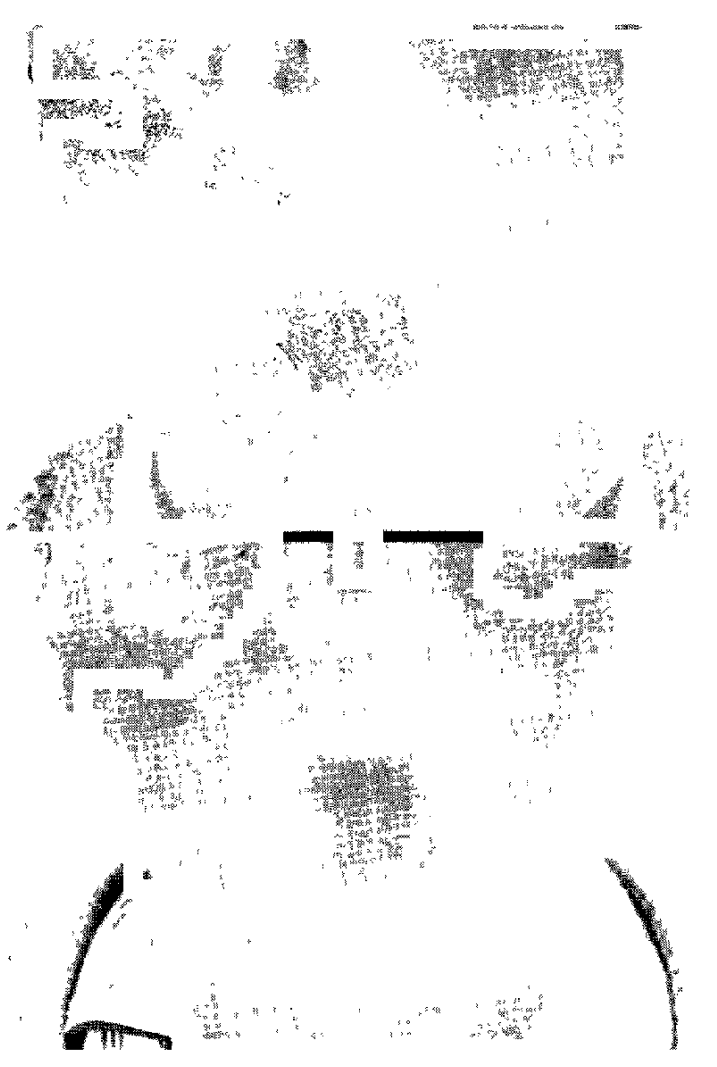

Fig. 1 Photographs showing deviation of tongue to the left at admission (upper), and at 6 weeks after onset when symptoms disappeared (lower).

may result from vascular lesions in the neck, such as aneurysm, dissection, or kinking of the external ICA. The mechanism of hypoglossal nerve paresis was apparently by nerve stretching and compression due to the abnormal vascular lesions. ${ }^{1,3,46}$ Intraoperative observation in one case found that the hypoglossal nerve was compressed by the tortuous ICA against the sternomastoid branch of the occipital artery. ${ }^{\text {6) }}$ Another case with focal enlargement and tortuosity of the ICA on MR imaging also supports this hypothesis. ${ }^{4)}$ In our case, the hypoglossal nerve paresis was the only neurological deficit, and dynamic changes were observed in the ICA on angiograms and MR images in the vicinity of the C1-2 vertebra. In this region, the hypoglossal nerve generally changes course and becomes superior to the ICA, below the posterior surface of the digastric muscle. The nerve then loops around the occipital branch of the external carotid artery. The cranial nerves and the sympathetic nerve do not adhere tightly to the ICA in this region. We think that local changes in the dissecting ICA was involved in the nerve paresis, and the kinking of the ICA may have
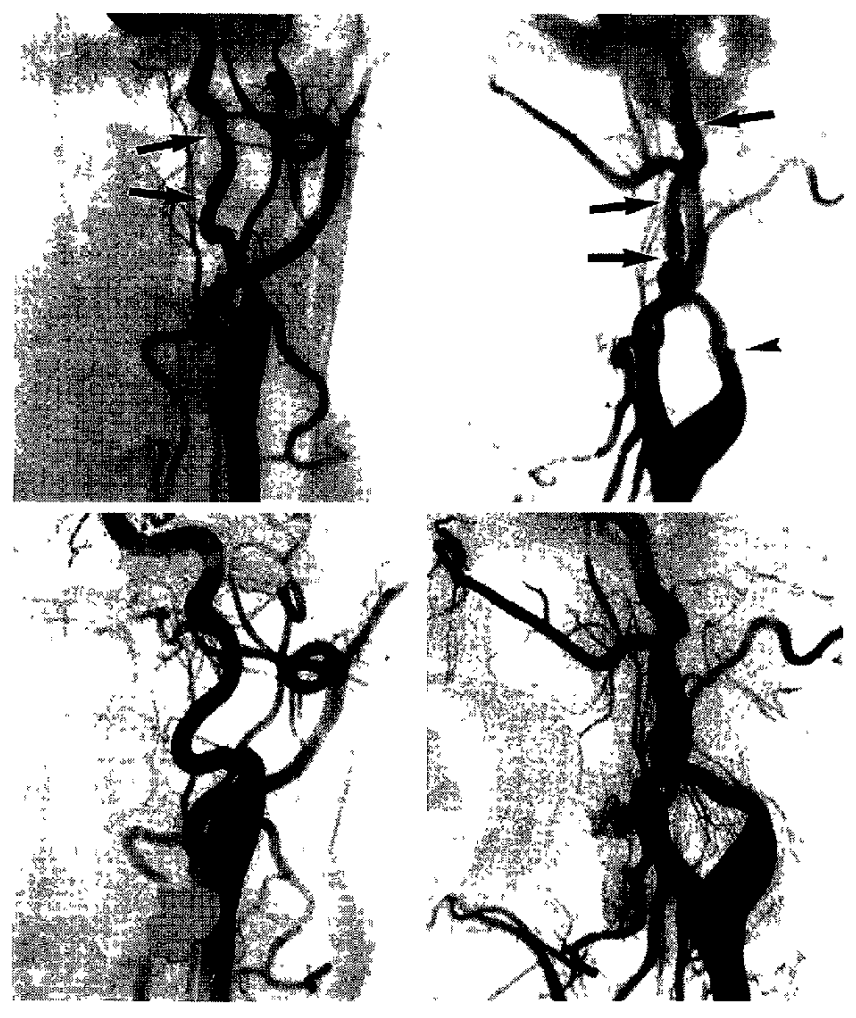

Fig. 2 Anteroposterior and lateral left carotid angiograms at admission (upper row) showing diffuse irregular narrowing (arrows) of the extracranial internal carotid artery and kinking (arrowhead) at the origin. Anteroposterior and lateral left carotid angiograms after disappearance of symptoms (lower row) showing only marked kinking, and the change in the course of the internal carotid artery.

been an additional factor.

The symptoms of extracranial nerve paresis due to ICA dissection usually improve or disappear spontaneously or after medical treatment, but other vascular lesions require surgical treatment. ${ }^{6)}$ Repeat MR imaging and angiography are needed for the correct diagnosis of ICA lesions in patients presenting with acute hypoglossal nerve paresis.

\section{References}

1] Farrell FW, Ellenberger $C$ : Transient hemilingual paralysis; Selective compression of the twelfth nerve and jugular bulb by a saccular carotid aneurysm. Neurology 22: 1061-1064, 1972

2) Fisher CM, Ojemann RG, Roberson GH: Spontaneous dissection of cervico-cerebral arteries. Can J Neurol Sci 5: 9-19, 1978

3) Goodmann JM, Zink WL, Cooper DF: Hemilingual 


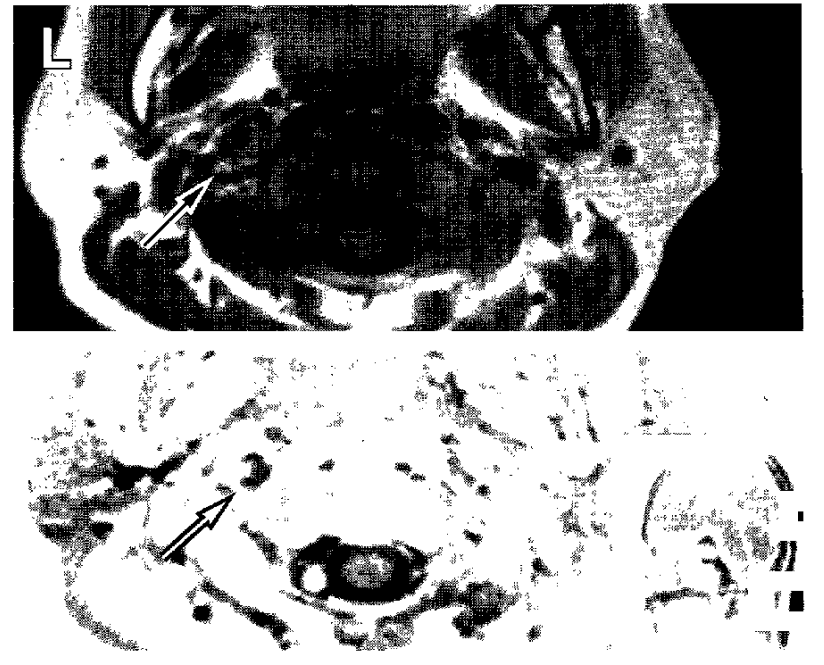

Fig. $3 \quad T_{1}$ - (upper) and $T_{2}$-weighted (lower) magnetic resonance images at admission showing a high intensity lesion (arrow) in the arterial wall of the left internal carotid artery.

paralysis caused by spontaneous carotid artery dissection. Arch Neurol 40: 653-654, 1983

4) Lieschke GJ, Davis S, Tress BM, Ebeling P: Spontaneous internal carotid artery dissection presenting as hypoglossal nerve palsy. Stroke 19: 1151-1155, 1988

5) Mokri B: Dissections of cervical and cephalic arteries, in Sundt TM (ed): Occlusive Cerebrovascular Disease. Philadelphia, WB Saunders, 1987, pp 38-59

6) Olivier A, Scotti G, Melancon D: Vascular entrapment of hypoglossal nerve in the neck. J Neurosurg 47:472475,1977

7) Saito $\mathrm{H}$, Onuma $\mathrm{T}$ : Isolated hypoglossal nerve palsy

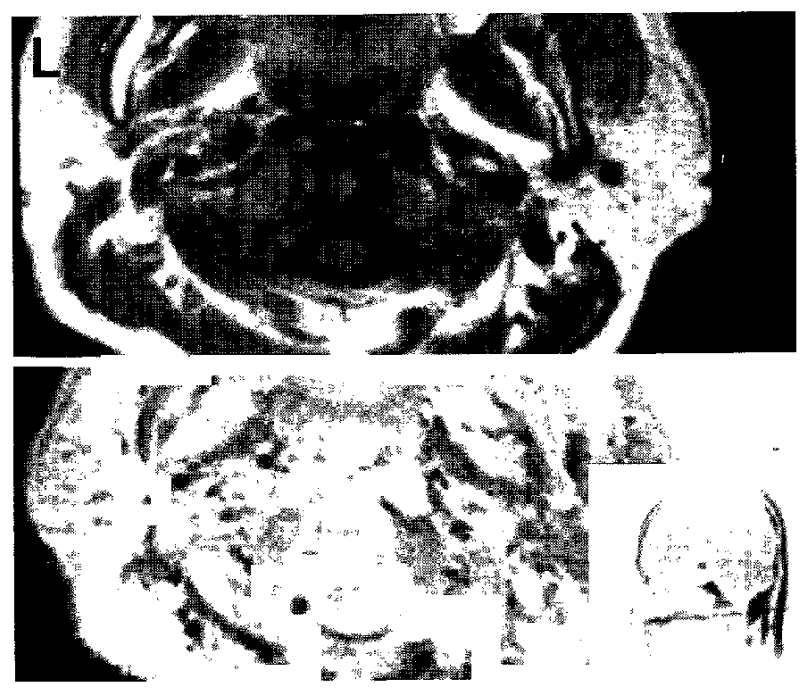

Fig. $4 T_{1}$ - (upper) and $T_{2}$-weighted (lower) magnetic resonance images at 6 weeks after onset showing disappearance of the high intensity lesions.

and Horner's syndrome with benign course. J Neurol Neurosurg Psychiatry 54: 282-283, 1991

8) Vanneste JAL, Davies G: Spontaneous dissection of the cervical internal carotid artery. Clin Neurol Neurosurg 86: 307-314, 1984

Address reprint requests to: S. Endo, M.D., Department of Neurosurgery, Toyama Medical and Pharmaceutical University, 2630 Sugitani, Toyama 930-0194, Japan. 